\title{
Modelo de Kirkwood para fluidos polares puros constituídos de moléculas com polarizabilidades desprezíveis
}

Kirkwood model for pure polar fluids of non-polarizable molecules

\author{
Paulo Peixoto*1@ Adonias Barros $\stackrel{10}{10}$, Mario Henrique Oliveira ${ }^{2}$ \\ ${ }^{1}$ Universidade Federal de Pernambuco, Núcleo de Formação Docente, Caruaru, PE, Brasil. \\ ${ }^{2}$ Universidade Federal Rural de Pernambuco, Unidade Acadêmica de Serra Talhada, Serra Talhada, PE, Brasil.
}

Recebido em 13 de junho de 2021. Revisado em 03 de julho de 2021. Aceito em 09 de julho de 2021.

\begin{abstract}
Neste artigo, guiamos o leitor ou a leitora à obtenção da equação de Kirkwood para fluidos polares puros de moléculas com polarizabilidades desprezíveis. Tal equação relaciona a constante dielétrica do fluido, sua densidade e sua temperatura ao momento de dipolo permanente de suas moléculas constituintes, e envolve ainda um fator adimensional $g$ - conhecido como fator de correlação de Kirkwood - cujo valor é uma expressão da correlação entre as orientações das moléculas do fluido, na situação de campo elétrico externo nulo. No percurso, buscamos evidenciar ao máximo a semelhança entre o modelo de Kirkwood e o modelo de Onsager aplicado a fluidos polares puros de moléculas não polarizáveis (ou com polarizabilidades desprezíveis).
\end{abstract}

Palavras-chave: modelo de Kirkwood, constante dielétrica, dielétricos.

In this paper, we guide the reader to obtain the Kirkwood equation for pure polar fluids of molecules with negligible polarizabilities. Such equation relates the dielectric constant of the fluid, its density and its temperature to the permanent dipole moment of its constituent molecules, and also involves a dimensionless factor $g$ - known as Kirkwood correlation factor - whose value is an expression of the correlation between the orientantions of the molecules of the fluid, in the situation of zero external electric field. Along the way, we seek to show as much as possible the similarity between the Kirkwood model and the Onsager model applied to pure polar fluids of non-polarizable molecules (or with negligible polarizabilities).

Keywords: Kirkwood model, dielectric constant, dielectrics.

\section{Introdução}

Em 1936, Lars Onsager obteve uma equação que relaciona a constante dielétrica $\epsilon_{r}$ de fluidos polares puros ao módulo $p_{0}$ do momento de dipolo permanente de suas moléculas constituintes, e também ao índice de refração $n$, à densidade numérica $N$ e à temperatura absoluta $T$ do meio [1]:1]

$$
\frac{\left(\epsilon_{r}-n^{2}\right)\left(2 \epsilon_{r}+n^{2}\right)}{\epsilon_{r}\left(n^{2}+2\right)^{2}}=\frac{N}{3 \epsilon_{0}} \frac{p_{0}^{2}}{3 k T}
$$

sendo $k$ a constante de Boltzmann e $\epsilon_{0}$ a permissividade elétrica do vácuo.

A equação de Onsager e as equações de Debye e de Clausius-Mossotti, que a precederam ${ }^{2}$ fazem parte dos

\footnotetext{
* Endereço de correspondência: phrpeixoto@yahoo.com.br

1 Neste artigo, escrevemos todas as equações no Sistema Internacional de Unidades (SI).

2 Sendo que a equação de Clausius-Mossotti se aplica apenas a fluidos apolares - ou seja, fluidos cujas moléculas têm momento de dipolo permanente nulo. As equações de Debye e de Onsager recaem na equação de Clausius-Mossotti, no caso de moléculas apolares.
}

modelos com aproximação de meio contínuo, em que uma única molécula do fluido é modelada como um dipolo em uma cavidade esférica microscópica, e além daquela cavidade o dielétrico é tratado como um meio contínuo (veja a Figura 1). Em um artigo recente [2], descrevemos esses modelos em detalhes, e dissemos que uma sequência natural, para os que se interessam por teoria de polarização elétrica, consiste no estudo do modelo de Kirkwood-Fröhlich, que de certa forma considera interações de curto alcance em fluidos polares puros, e foi desenvolvido - inicialmente por Kirkwood [3], tendo recebido depois a contribuição de Fröhlich [4] - a partir do modelo de Onsager.

A equação de Kirkwood-Fröhlich 3

$$
\frac{\left(\epsilon_{r}-n^{2}\right)\left(2 \epsilon_{r}+n^{2}\right)}{\epsilon_{r}\left(n^{2}+2\right)^{2}}=\frac{N}{3 \epsilon_{0}} \frac{p_{0}^{2}}{3 k T} g
$$

\footnotetext{
${ }^{3}$ A equação de Kirkwood-Fröhlich também é apresentada com $\epsilon_{\infty}$ - a chamada constante dielétrica característica para a polarização induzida - no lugar de $n^{2}$. Mas $\epsilon_{\infty}=n^{2}$, como explicamos em nosso artigo anterior [2], no trecho em que introduzimos o índice de refração na equação de Onsager (sendo que, lá, não usamos a notação " $\epsilon_{\infty}$ ").
} 


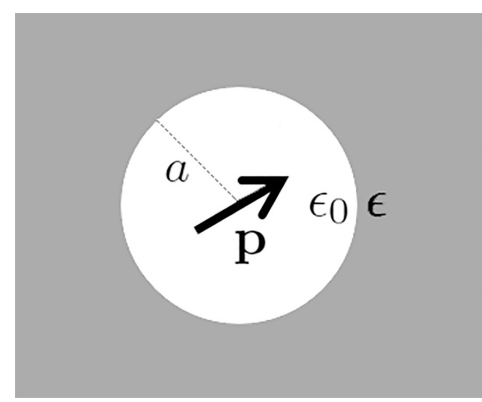

Figura 1: Cavidade esférica microscópica imaginária, de raio $a$, em um fluido dielétrico (nos modelos com aproximação de meio contínuo). A cavidade contém uma única molécula do fluido, aqui representada por seu momento de dipolo total $\mathbf{p}$ (em um determinado instante). Além da cavidade, o dielétrico é modelado como um meio contínuo de permissividade $\epsilon$.

difere da equação de Onsager pela presença do fator adimensional $g$, denominado fator de correlação de Kirkwood (cujo valor é uma expressão da correlação entre as orientações das moléculas do fluido, na situação de campo elétrico externo nulo), e é uma extensão da equação de Kirkwood,

$$
\frac{\left(\epsilon_{r}-1\right)\left(2 \epsilon_{r}+1\right)}{9 \epsilon_{r}}=\frac{N}{3 \epsilon_{0}} \frac{p_{0}^{2}}{3 k T} g,
$$

que se aplica apenas a fluidos polares puros de moléculas não polarizáveis (ou com polarizabilidades desprezíveis). O índice de refração de um meio constituído de moléculas não polarizáveis é igual ao índice de refração do vácuo ${ }^{4}$ e fazendo $n=1$ na equação de Kirkwood-Fröhlich obtemos a equação de Kirkwood. Nesse sentido, a equação de Kirkwood pode ser vista como um caso particular da equação de Kirkwood-Fröhlich, mas, historicamente, primeiro surgiu a equação de Kirkwood. A consideração de polarizabilidades moleculares não nulas no desenvolvimento apresentado por Kirkwood envolve dificuldades técnicas que foram, posteriormente, contornadas por Fröhlich, de modo um tanto criativo. Mas, neste texto, estaremos interessados apenas na equação de Kirkwood, e guiaremos o leitor ou a leitora à sua obtenção.

Organizamos nossa apresentação buscando evidenciar ao máximo a semelhança entre o modelo de Kirkwood e o modelo de Onsager aplicado a fluidos polares puros de moléculas não polarizáveis (ou com polarizabilidades desprezíveis). Acreditamos que isso contribui para uma melhor compreensão geral desses modelos.

Para evitarmos repetições que levariam a um aumento considerável do tamanho deste artigo, admitiremos que você leu nosso artigo anterior [2] (e está com ele à mão). Se não leu, e tem interesse no modelo de Kirkwood, saiba que a primeira frase do artigo de Kirkwood [3] (no resumo) é: "An extension of the Onsager theory of

\footnotetext{
$\overline{4}$ Faça $\alpha=0$ na Eq. (39) do nosso artigo anterior 2 - a saber $\left(n^{2}-1\right) /\left(n^{2}+2\right)=N \alpha / 3 \epsilon_{0}-$ e você obterá $n=1$.
}

dielectric polarization is presented." Você esperaria compreender aquele artigo sem nenhum conhecimento prévio sobre o modelo de Onsager? Veja, não estamos dizendo que seria impossível apresentar o modelo de Kirkwood para um público que não conhece o modelo de Onsager, mas esse provavelmente seria um caminho muito mais árduo para todos. Então, se você não é um especialista em teoria de polarização elétrica, tem interesse na área, e não leu nosso artigo anterior, sugerimos fortemente que o leia antes de fazer uma leitura minuciosa deste artigo.

\section{Obtendo a Equação de Onsager para o Caso Particular de Moléculas não Polarizáveis}

Em nossa jornada para obtenção da equação de Kirkwood para fluidos polares puros constituídos de moléculas não polarizáveis (ou com polarizabilidades desprezíveis), inicialmente obteremos a equação de Onsager para o caso particular de moléculas não polarizáveis. É claro, se tivéssemos interesse apenas na equação final, bastaria fazermos $n=1$ na equação de Onsager, e obteríamos (compare com a equação de Kirkwood)

$$
\frac{\left(\epsilon_{r}-1\right)\left(2 \epsilon_{r}+1\right)}{9 \epsilon_{r}}=\frac{N}{3 \epsilon_{0}} \frac{p_{0}^{2}}{3 k T},
$$

que é a equação de Onsager para o caso particular de fluidos polares puros constituídos de moléculas não polarizáveis (ou com polarizabilidades desprezíveis). Mas o que nos interessa, principalmente, é o desenvolvimento do modelo!

Pois bem, se $\alpha=0$, a sequência de quatro passos apresentada em nosso artigo anterior [2], para a obtenção da equação de Onsager, pode ser simplificada para:

- Passo $\mathbf{1}^{\prime}$ : Usando mecânica estatística, calcule $\overline{\mathbf{p}_{\mathbf{0}}}$ para a molécula na cavidade microscópica da Fig. 1 $\left(\operatorname{com} \mathbf{p}=\mathbf{p}_{\mathbf{0}}\right)$, e então obtenha

$$
\mathbf{P}=N \overline{\mathbf{p}_{\mathbf{0}}}
$$

em que $\mathbf{P}$ é o vetor polarização elétrica e $N$ é o número de moléculas por unidade de volume do fluido.

- Passo 2': Substitua o módulo de $\mathbf{P}$ (que será proporcional a $E$ ) na igualdade

$$
\epsilon_{r}=1+\frac{P}{\epsilon_{0} E}
$$

Para o cálculo de $\overline{\mathbf{p}_{\mathbf{0}}}$ precisaremos obter uma expressão para o campo interno $\mathbf{E}_{\text {int }}$ que age sobre a molécula da Fig. 1. com $\mathbf{p}=\mathbf{p}_{\mathbf{0}}$, mas com a eliminação do passo 2 na sequência de passos de nosso artigo anterior, o passo 1 daquela sequência não precisa mais ficar explícito. O cálculo de $\mathbf{E}_{\mathbf{i n t}}$ já foi realizado em nosso 
artigo anterior (com $\mathbf{p}$ não necessariamente igual a $\mathbf{p}_{\mathbf{0}}$ ), então basta trocarmos $\mathbf{p}$ por $\mathbf{p}_{\mathbf{0}}$ para obtermos:

$$
\mathbf{E}_{\text {int }}=\mathbf{G}+\mathbf{R},
$$

com

$$
\mathbf{G}=\left(\frac{3 \epsilon_{r}}{2 \epsilon_{r}+1}\right) \mathbf{E}
$$

e

$$
\mathbf{R}=\left(\frac{\epsilon_{r}-1}{2 \epsilon_{r}+1}\right) \frac{\mathbf{p}_{\mathbf{0}}}{2 \pi \epsilon_{0} a^{3}} .
$$

Podemos simplificar as coisas ainda mais, e reunir os dois passos acima em um só:

- Usando mecânica estatística, calcule $\overline{\mathbf{p}_{\mathbf{0}}}$ para a molécula na cavidade microscópica da Fig. 11 (com $\left.\mathbf{p}=\mathbf{p}_{\mathbf{0}}\right)$, e substitua a expressão obtida na igualdade

$$
\epsilon_{0}\left(\epsilon_{r}-1\right) E=N \overline{\mathbf{p}_{\mathbf{0}}} \cdot \hat{\mathbf{z}}
$$

em que $\hat{\mathbf{z}}$ é o versor com a direção e o sentido do campo $\mathbf{E} .^{5}$

Calcularemos $\overline{\mathbf{p}_{\mathbf{0}}}$ centrando nossa atenção, como antes [2], na molécula da Fig. 1] (mas agora com $\mathbf{p}=\mathbf{p}_{\mathbf{0}}$ ), e consideraremos o restante do fluido (o meio contínuo além da cavidade microscópica) como um reservatório térmico, e, portanto, trabalharemos com o ensemble canônico da mecânica estatística. Temos, então:

$$
\overline{\mathbf{p}_{\mathbf{0}}}=\frac{\sum_{r}\left(\mathbf{p}_{\mathbf{0}}\right)_{r} e^{-\beta \varepsilon_{r}}}{\sum_{r} e^{-\beta \varepsilon_{r}}},
$$

em que $\varepsilon_{r}$ é a energia de um determinado estado $r$ da molécula da Fig. 1 ( $\left.\operatorname{com} \mathbf{p}=\mathbf{p}_{\mathbf{0}}\right)$, e os somatórios são realizados sobre todos os estados acessíveis àquela molécula. Como sempre, $\beta=1 / k T$.

Separando a energia $\varepsilon_{r}$ em seus termos de energia cinética $t_{r}$ e energia potencial $u_{r}$, realizando a fatoração usual

$$
e^{-\beta \varepsilon_{r}}=e^{-\beta\left(t_{r}+u_{r}\right)}=e^{-\beta t_{r}} e^{-\beta u_{r}},
$$

e lembrando que o estado $r$ é determinado por coordenadas generalizadas $q_{1}, q_{2}, \ldots$ e por seus momentos associados $p_{1}, p_{2}, \ldots$, sendo que $t_{r}=t_{p_{1}, p_{2}, \ldots} \equiv t_{p}$, $u_{r}=u_{q_{1}, q_{2}, \ldots} \equiv u_{q}$ e $\left(\mathbf{p}_{\mathbf{0}}\right)_{r}=\left(\mathbf{p}_{\mathbf{0}}\right)_{q_{1}, q_{2}, \ldots} \equiv\left(\mathbf{p}_{\mathbf{0}}\right)_{q}$, podemos reescrever a igualdade (5) como

$$
\begin{aligned}
\overline{\mathbf{p}_{\mathbf{0}}} & =\frac{\sum_{p} \sum_{q}\left(\mathbf{p}_{\mathbf{0}}\right)_{q} e^{-\beta t_{p}} e^{-\beta u_{q}}}{\sum_{p} \sum_{q} e^{-\beta t_{p}} e^{-\beta u_{q}}} \\
& =\frac{\left(\sum_{p} e^{-\beta t_{p}}\right)\left(\sum_{q}\left(\mathbf{p}_{\mathbf{0}}\right)_{q} e^{-\beta u_{q}}\right)}{\left(\sum_{p} e^{-\beta t_{p}}\right)\left(\sum_{q} e^{-\beta u_{q}}\right)} .
\end{aligned}
$$

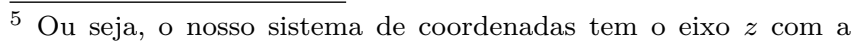
mesma direção e o mesmo sentido que o campo $\mathbf{E}$.
}

Podemos usar o próprio vetor $\mathbf{p}_{\mathbf{0}}$ para especificar a configuração da molécula da Fig. 1] ( com $\mathbf{p}=\mathbf{p}_{\mathbf{0}}$, vale sempre lembrar), e, portanto, podemos reescrever a igualdade 6 como

$$
\overline{\mathbf{p}_{\mathbf{0}}}=\frac{\sum_{\mathbf{p}_{\mathbf{0}}} \mathbf{p}_{\mathbf{0}} e^{-\beta u_{\mathbf{p}_{\mathbf{0}}}}}{\sum_{\mathbf{p}_{\mathbf{0}}} e^{-\beta u_{\mathbf{p}_{\mathbf{0}}}}},
$$

significando que os somatórios são efetuados sobre todas as orientações possíveis para $\mathbf{p}_{\mathbf{0}}$. É claro, como os ângulos $\theta$ e $\phi$ que determinam, em coordenadas esféricas, a orientação de $\mathbf{p}_{\mathbf{0}}$ são variáveis contínuas, esses somatórios devem ser substituídos por integrais nessas variáveis. Mas não precisaremos nos ocupar com isso agora.

Vamos obter uma expressão para $u_{\mathbf{p}_{\mathbf{0}}}$. Estamos considerando que a molécula na cavidade esférica microscópica da Fig. 11é suficientemente representada por um dipolo rígido com momento de dipolo $\mathbf{p}_{\mathbf{0}}$, submetido ao campo $\mathbf{E}_{\text {int }}=\mathbf{G}+\mathbf{R}$ (veja as igualdades (1), (2) e (3)). Mas como $\mathbf{R}$ é paralelo a $\mathbf{p}_{\mathbf{0}}$, e como a molécula é não polarizável, o campo de reação $\mathbf{R}$ não tem qualquer efeito sobre aquela molécula - ou seja, ele não produz nela uma polarização adicional, nem exerce sobre ela torque algum. Por outro lado, G é um campo uniforme. Então temos para $u_{\mathbf{p}_{\mathbf{0}}}$ a expressão da energia potencial de um dipolo rígido submetido a um campo elétrico uniforme: $u_{\mathbf{p}_{\mathbf{0}}}=-\mathbf{p}_{\mathbf{0}} \cdot \mathbf{G}$. Portanto, usando a expressão para $\mathbf{G}$ (veja a igualdade (2) obtemos

$$
u_{\mathbf{p}_{\mathbf{0}}}=-\left(\frac{3 \epsilon_{r}}{2 \epsilon_{r}+1}\right) \mathbf{p}_{\mathbf{0}} \cdot \mathbf{E},
$$

que podemos reescrever como

$$
u_{\mathbf{p}_{\mathbf{0}}}=-\xi^{\prime} \mathbf{p}_{\mathbf{0}} \cdot \mathbf{E},
$$

com

$$
\xi^{\prime} \equiv \frac{3 \epsilon_{r}}{2 \epsilon_{r}+1} .
$$

Substituindo (8) em (7) obtemos:

$$
\overline{\mathbf{p}_{\mathbf{0}}}=\frac{\sum_{\mathbf{p}_{\mathbf{o}}} \mathbf{p}_{\mathbf{0}} e^{\beta \xi^{\prime} \mathbf{p}_{\mathbf{0}} \cdot \mathbf{E}}}{\sum_{\mathbf{p}_{\mathbf{0}}} e^{\beta \xi^{\prime} \mathbf{p}_{\mathbf{0}} \cdot \mathbf{E}}},
$$

com $\xi^{\prime}$ dado em 9 .

Observe que usando a igualdade $u_{\mathbf{p}_{\mathbf{0}}}=-\mathbf{p}_{\mathbf{0}} \cdot \mathbf{E}_{\mathbf{i n t}}$, em vez de $u_{\mathbf{p}_{\mathbf{0}}}=-\mathbf{p}_{\mathbf{0}} \cdot \mathbf{G}$, obteríamos

$$
\begin{aligned}
u_{\mathbf{p}_{\mathbf{0}}} & =-\mathbf{p}_{\mathbf{0}} \cdot \mathbf{E}_{\mathbf{i n t}}=-\mathbf{p}_{\mathbf{0}} \cdot \mathbf{G}-\mathbf{p}_{\mathbf{0}} \cdot \mathbf{R} \\
& =-\left(\frac{3 \epsilon_{r}}{2 \epsilon_{r}+1}\right) \mathbf{p}_{\mathbf{0}} \cdot \mathbf{E}-\left(\frac{\epsilon_{r}-1}{2 \epsilon_{r}+1}\right) \frac{p_{0}^{2}}{2 \pi \epsilon_{0} a^{3}} .
\end{aligned}
$$

Mas este último termo é constante, e, portanto, sem qualquer significado físico - já que o que importam são as variações da energia potencial, e não seu valor absoluto. De fato, a substituição em (7) desta última expressão para $u_{\mathbf{p}_{\mathbf{0}}}$ resultaria, após uma simplificação, 
na mesma expressão obtida em 10 (verifique). É outra forma de ver que o campo de reação não desempenha papel relevante no modelo de Onsager aplicado a fluidos polares puros de moléculas não polarizáveis.

O desenvolvimento a partir da igualdade 10 é idêntico ao apresentado em nosso artigo anterior [2], entre as igualdades (30) e (35) do mesmo - apenas trocando-se $\xi$ por $\xi^{\prime}$, é claro. É importante que você releia aquele trecho. Observe, particularmente, que o cálculo de $\overline{\mathbf{p}_{\mathbf{0}}}$ inicialmente nos levou a (agora trocando $\xi$ por $\left.\xi^{\prime}\right)$

$$
\overline{\mathbf{p}}_{\mathbf{0}}=p_{0} \hat{\mathbf{z}}\left(\operatorname{coth}\left(\xi^{\prime} p_{0} E / k T\right)-\frac{1}{\xi^{\prime} p_{0} E / k T}\right),
$$

e apenas para $\xi^{\prime} p_{0} E / k T \ll 1$ temos, como uma ótima aproximação,

$$
\overline{\mathbf{p}_{\mathbf{0}}} \approx \xi^{\prime} \frac{p_{0}^{2}}{3 k T} \mathbf{E} .
$$

Substituindo (12) em (4) (fazendo uso da expressão para $\xi^{\prime}$ em (9) ) obtemos, após uma pequena manipulação algébrica,

$$
\frac{\left(\epsilon_{r}-1\right)\left(2 \epsilon_{r}+1\right)}{9 \epsilon_{r}}=\frac{N}{3 \epsilon_{0}} \frac{p_{0}^{2}}{3 k T}
$$

que é a equação de Onsager para o caso particular de fluidos polares puros constituídos de moléculas não polarizáveis (ou com polarizabilidades desprezíveis).

\subsection{Desenvolvimento alternativo}

Agora, vamos apresentar um desenvolvimento, entre as igualdades (4) e (13), que se assemelha bastante ao desenvolvimento que irá nos levar, na próxima seção, à equação de Kirkwood. A mudança consiste essencialmente em supormos, logo de início, que a relação entre $\overline{\mathbf{p}_{\mathbf{0}}}$ e $\mathbf{E}$ é linear para campos de intensidade suficientemente baixa (em vez de realizarmos o cálculo de $\overline{\mathbf{p}_{\mathbf{0}}}$ para o caso geral, como fizemos acima, considerando apenas ao final $\xi^{\prime} p_{0} E / k T \ll 1$ e obtendo, com isso, uma relação linear entre $\overline{\mathbf{p}_{\mathbf{0}}}$ e $\left.\mathbf{E}\right)$. Por um lado, tratase de uma ideia atraente; afinal, se o que nos interessa é uma relação linear entre $\overline{\mathbf{p}_{\mathbf{0}}}$ e $\mathbf{E}$, por que não partir da hipótese de que $\overline{\mathbf{p}_{\mathbf{0}}}$ é proporcional a $\mathbf{E}$ para intensidades de campo suficientemente baixas? Por outro lado, como teríamos certeza de que obteríamos, com a realização do cálculo de $\overline{\mathbf{p}_{\mathbf{0}}}$ para o caso geral, uma relação linear entre $\overline{\mathbf{p}_{\mathbf{0}}}$ e $\mathbf{E}$, no caso de campos de baixa intensidade? Afinal, alguns sistemas são intrinsecamente não-lineares. Mas como já realizamos o cálculo de $\overline{\mathbf{p}_{\mathbf{0}}}$ para o caso geral, e obtivemos que $\overline{\mathbf{p}_{\mathbf{0}}}$ é proporcional a $\mathbf{E}$ se $\xi^{\prime} p_{0} E / k T \ll 1$, podemos seguir com segurança por essa via alternativa ${ }^{6}$

\footnotetext{
6 É claro, se no caso geral obtivéssemos, mesmo em primeira aproximação, uma relação intrinsecamente não linear entre $\overline{\mathbf{p}_{\mathbf{0}}}$ e $\mathbf{E}$ (como, por exemplo, $\left.\overline{\mathbf{p o}} \propto E^{2} \mathbf{E}\right)$, em certo sentido isso
}

Pois bem, expandindo $\overline{\mathbf{p}_{\mathbf{0}}} \cdot \hat{\mathbf{z}}$ (lembrando que $\hat{\mathbf{z}}$ é o versor com a direção e o sentido do campo E) em potências de $E(E=\mathbf{E} \cdot \hat{\mathbf{z}})$ de expoentes não negativos, a partir de $E=0$, e tomando apenas os dois primeiros termos (considerando que a intensidade $E$ do campo macroscópico $\mathbf{E}$ é suficientemente baixa), obtemos

$$
\left(\overline{\mathbf{p}_{\mathbf{0}}} \cdot \hat{\mathbf{z}}\right)(E) \approx\left(\overline{\mathbf{p}_{\mathbf{0}}} \cdot \hat{\mathbf{z}}\right)(0)+\left.\frac{\partial\left(\overline{\mathbf{p}_{\mathbf{0}}} \cdot \hat{\mathbf{z}}\right)}{\partial E}\right|_{E=0} E .
$$

Mas, é claro, para $E=0$ temos $\overline{\mathbf{p}_{\mathbf{0}}}=0$, e, portanto (a partir daqui usando "=", em vez de “ $\approx "$ ),

$$
\overline{\mathbf{p}_{\mathbf{0}}} \cdot \hat{\mathbf{z}}=\left.\frac{\partial\left(\overline{\mathbf{p}_{\mathbf{0}}} \cdot \hat{\mathbf{z}}\right)}{\partial E}\right|_{E=0} E .
$$

Esta última igualdade corresponde a

$$
\overline{\mathbf{p}_{\mathbf{0}}}=\left.\frac{\partial\left(\overline{\mathbf{p}_{\mathbf{0}}} \cdot \hat{\mathbf{z}}\right)}{\partial E}\right|_{E=0} \mathbf{E},
$$

que é a primeira versão para a relação de proporcionalidade que buscamos entre $\overline{\mathbf{p}_{\mathbf{0}}}$ e $\mathbf{E}$. Obviamente, queremos chagar à igualdade (12), mas por outro caminho (que será útil para a compreensão do desenvolvimento que levará à equação de Kirkwood, na próxima seção).

Faremos no segundo membro da igualdade (14) - mais especificamente em $\left.\left[\partial\left(\overline{\mathbf{p}_{\mathbf{0}}} \cdot \hat{\mathbf{z}}\right) / \partial E\right]\right|_{E=0}-$ uma mudança de variável: de $E$ para

$$
G=\left(\frac{3 \epsilon_{r}}{2 \epsilon_{r}+1}\right) E
$$

(veja a igualdade (2)). Pela aplicação da regra da cadeia, obtemos

$$
\left.\frac{\partial\left(\overline{\mathbf{p}_{\mathbf{0}}} \cdot \hat{\mathbf{z}}\right)}{\partial E}\right|_{E=0}=\left.\frac{\partial\left(\overline{\mathbf{p}_{\mathbf{0}}} \cdot \hat{\mathbf{z}}\right)}{\partial G}\right|_{G=0} \underbrace{\left.\frac{\partial G}{\partial E}\right|_{E=0}}_{3 \epsilon_{r} /\left(2 \epsilon_{r}+1\right)},
$$

e então podemos reescrever a igualdade 14 como

$$
\overline{\mathbf{p}_{\mathbf{0}}} \cdot \hat{\mathbf{z}}=\left.\frac{\partial\left(\overline{\mathbf{p}_{\mathbf{0}}} \cdot \hat{\mathbf{z}}\right)}{\partial G}\right|_{G=0}\left(\frac{3 \epsilon_{r}}{2 \epsilon_{r}+1}\right) E .
$$

Esta mudança de variável se justifica porque o cálculo de $\overline{\mathbf{p}_{\mathbf{0}}}$ envolve $\mathbf{E}_{\mathbf{i n t}}-$ mais especificamente $\left.\mathbf{G}\right]^{7}$-, em vez de $\mathbf{E}$.

Convém, neste ponto, combinarmos as igualdades (4) e (15), e com isso obtemos:

$$
\frac{\left(\epsilon_{r}-1\right)\left(2 \epsilon_{r}+1\right)}{9 \epsilon_{r}}=\left.\frac{N}{3 \epsilon_{0}} \frac{\partial\left(\overline{\mathbf{p}_{\mathbf{0}}} \cdot \hat{\mathbf{z}}\right)}{\partial G}\right|_{G=0} .
$$

comprometeria o modelo como um todo, pois já na igualdade (4) uma relação linear entre $\overline{\mathbf{p o}}$ e $\mathbf{E}$ é esperada (para campos de baixa intensidade). Contudo, essa expectativa não garante que o desenvolvimento nos daria, ao final, uma relação linear entre aquelas duas grandezas. Tudo isso deve ser bem compreendido, e faz parte da discussão inesgotável do que é modelagem em física. 7 Lembre-se que o campo de reação $\mathbf{R}$ não tem qualquer efeito sobre uma molécula não polarizável. 
Para verificar a consistência de nosso desenvolvimento até este ponto, substitua (12) (observando que $\left.\xi^{\prime} E=G\right)$ em $(16)$, e verifique que com isso obtemos a equação de Onsager para o caso particular de fluidos polares puros constituídos de moléculas não polarizáveis (ou com polarizabilidades desprezíveis) ou seja, a equação (13). Mas, é claro, não faz parte desse desenvolvimento alternativo o uso da expressão para $\overline{\mathbf{p}_{\mathbf{0}}}$ em $(12)$. O que faremos a seguir será trabalhar a quantidade $\left.\left[\partial\left(\overline{\mathbf{p}_{\mathbf{0}}} \cdot \hat{\mathbf{z}}\right) / \partial G\right]\right|_{G=0}$ sem a realização de um cálculo explícito de $\overline{\mathbf{p}_{\mathbf{0}}}$ - que é o que estamos evitando 8

Tomando o produto interno, com $\hat{\mathbf{z}}$, de ambos os membros da igualdade (10) (reescrita de forma ligeiramente diferente) obtemos

$$
\overline{\mathbf{p}_{\mathbf{0}}} \cdot \hat{\mathbf{z}}=\frac{1}{Z} \sum_{\mathbf{p}_{\mathbf{0}}}\left(\mathbf{p}_{\mathbf{0}} \cdot \hat{\mathbf{z}}\right) e^{-\beta u_{\mathbf{p}_{\mathbf{o}}}},
$$

com

$$
Z \equiv \sum_{\mathbf{p}_{\mathbf{0}}} e^{-\beta u_{\mathbf{p}_{\mathbf{0}}}}
$$

e (veja a igualdade (8), e lembre-se que $\xi^{\prime} \mathbf{E}=\mathbf{G}$ )

$$
u_{\mathbf{p}_{\mathbf{o}}}=-\mathbf{p}_{\mathbf{0}} \cdot \mathbf{G}=-\left(\mathbf{p}_{\mathbf{0}} \cdot \hat{\mathbf{z}}\right) G=-\left(p_{0} \cos \theta\right) G,
$$

em que $\theta$ é o ângulo entre $\mathbf{p}_{\mathbf{0}}$ e $\hat{\mathbf{z}}$ (ou, equivalentemente, entre $\mathbf{p}_{\mathbf{0}}$ e $\mathbf{G}$ ). Estamos escrevendo a expressão para $\overline{\mathbf{p}_{\mathbf{0}}} \cdot \hat{\mathbf{z}}$ em (17) daquela forma buscando um máximo de semelhança entre este desenvolvimento e o que será apresentado na próxima seção.

A partir de (17), obtemos:

$$
\begin{aligned}
\left.\frac{\partial\left(\overline{\mathbf{p}_{\mathbf{0}}} \cdot \hat{\mathbf{z}}\right)}{\partial G}\right|_{G=0}= & \left.\left(-\frac{1}{Z^{2}} \frac{\partial Z}{\partial G} \sum_{\mathbf{p}_{\mathbf{0}}}\left(\mathbf{p}_{\mathbf{0}} \cdot \hat{\mathbf{z}}\right) e^{-\beta u_{\mathbf{p}_{\mathbf{0}}}}\right)\right|_{G=0} \\
& +\left.\left(\frac{1}{Z} \sum_{\mathbf{p}_{\mathbf{0}}} \frac{\partial}{\partial G}\left[\left(\mathbf{p}_{\mathbf{0}} \cdot \hat{\mathbf{z}}\right) e^{-\beta u_{\mathbf{p}}}\right]\right)\right|_{G=0} .
\end{aligned}
$$

Vamos mostrar que o primeiro dos dois termos no segundo membro, acima, é nulo:

$$
\begin{aligned}
& \left.\left(-\frac{1}{Z^{2}} \frac{\partial Z}{\partial G} \sum_{\mathbf{p}_{\mathbf{0}}}\left(\mathbf{p}_{\mathbf{0}} \cdot \hat{\mathbf{z}}\right) e^{-\beta u_{\mathbf{p}_{\mathbf{0}}}}\right)\right|_{G=0} \\
& =\left.\left(-\frac{1}{Z} \frac{\partial Z}{\partial G} \frac{1}{Z} \sum_{\mathbf{p}_{\mathbf{0}}}\left(\mathbf{p}_{\mathbf{0}} \cdot \hat{\mathbf{z}}\right) e^{-\beta u_{\mathbf{p}_{\mathbf{0}}}}\right)\right|_{G=0}
\end{aligned}
$$

\footnotetext{
8 Esta nota de rodapé destina-se aos leitores que podem ter levantado a seguinte questão: no teste de consistência acima, não teria sido mais adequado substituirmos em (16) não a expressão para $\overline{\mathbf{p}_{\mathbf{0}}}$ em $[12$, mas aquela em 11 ? De fato, mas a confusão que pode surgir ao fazermos isto é que embora $L(x) \equiv \operatorname{coth} x-1 / x$ possua derivada para $x=0$, as funções $\operatorname{coth} x$ e $1 / x$ não possuem, e, com isso, não podemos aplicar a regra da diferença para o cálculo de derivadas. Sabemos que $L(x)$ - a chamada função de Langevin - possui derivada para $x=0$ porque $\operatorname{coth} x$ pode ser expandida em série (de Laurent) como coth $x=1 / x+x / 3-x^{3} / 45+2 x^{5} / 945-$ $x^{7} / 4725+\cdots$, e o termo $1 / x$, que faz com que $\operatorname{coth} x$ não tenha derivada para $x=0$, é cancelado na diferença $\operatorname{coth} x-1 / x$, que define $L(x)$.
}

$$
\begin{aligned}
& =\left.\left(-\frac{1}{Z} \frac{\partial Z}{\partial G} \overline{\mathbf{p}_{\mathbf{0}} \cdot \hat{\mathbf{z}}}\right)\right|_{G=0} \\
& =\left.\left.\left(-\frac{1}{Z} \frac{\partial Z}{\partial G}\right)\right|_{G=0}\left(\overline{\mathbf{p}_{\mathbf{0}} \cdot \hat{\mathbf{z}}}\right)\right|_{G=0}=0,
\end{aligned}
$$

pois

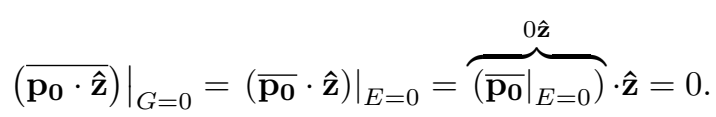

Segue então que

$$
\left.\frac{\partial\left(\overline{\mathbf{p}_{\mathbf{0}}} \cdot \hat{\mathbf{z}}\right)}{\partial G}\right|_{G=0}=\left.\left(\frac{1}{Z} \sum_{\mathbf{p}_{\mathbf{0}}} \frac{\partial}{\partial G}\left[\left(\mathbf{p}_{\mathbf{0}} \cdot \hat{\mathbf{z}}\right) e^{-\beta u_{\mathbf{p}_{\mathbf{0}}}}\right]\right)\right|_{G=0} .
$$

Temos, no segundo membro da igualdade acima:

$$
\begin{aligned}
\frac{\partial}{\partial G}\left[\left(\mathbf{p}_{\mathbf{0}} \cdot \hat{\mathbf{z}}\right) e^{\left.-\beta u_{\mathbf{p}_{\mathbf{0}}}\right]=}\right. & {\left[\frac{\partial}{\partial G}\left(\mathbf{p}_{\mathbf{0}} \cdot \hat{\mathbf{z}}\right)\right] e^{-\beta u_{\mathbf{p}_{\mathbf{0}}}} } \\
& +\left(\mathbf{p}_{\mathbf{0}} \cdot \hat{\mathbf{z}}\right) e^{-\beta u_{\mathbf{p}_{\mathbf{0}}}}\left[-\beta \frac{\partial u_{\mathbf{p}_{\mathbf{0}}}}{\partial G}\right]
\end{aligned}
$$

mas

$$
\frac{\partial}{\partial G}\left(\mathbf{p}_{\mathbf{0}} \cdot \hat{\mathbf{z}}\right)=\left(\frac{\partial \mathbf{p}_{\mathbf{0}}}{\partial G}\right) \cdot \hat{\mathbf{z}}=0,
$$

pois $\mathbf{p}_{\mathbf{0}}$ não é função de $G !^{9}$ Segue que

$$
\left.\frac{\partial\left(\overline{\mathbf{p}_{\mathbf{0}}} \cdot \hat{\mathbf{z}}\right)}{\partial G}\right|_{G=0}=-\left.\beta\left(\frac{1}{Z} \sum_{\mathbf{p}_{\mathbf{0}}}\left(\mathbf{p}_{\mathbf{0}} \cdot \hat{\mathbf{z}}\right) \frac{\partial u_{\mathbf{p}_{\mathbf{o}}}}{\partial G} e^{-\beta u_{\mathbf{p}_{\mathbf{0}}}}\right)\right|_{G=0}
$$

e, como $\partial u_{\mathbf{p}_{\mathbf{0}}} / \partial G=-\left(\mathbf{p}_{\mathbf{0}} \cdot \hat{\mathbf{z}}\right)=-p_{0} \cos \theta \quad($ veja a igualdade (19), obtemos

$$
\begin{aligned}
\left.\frac{\partial\left(\overline{\mathbf{p}_{\mathbf{0}}} \cdot \hat{\mathbf{z}}\right)}{\partial G}\right|_{G=0} & =\left.\beta(\frac{1}{Z} \sum_{\mathbf{p}_{\mathbf{0}}} \overbrace{\left(\mathbf{p}_{\mathbf{0}} \cdot \hat{\mathbf{z}}\right)^{2}}^{\left(p_{0} \cos \theta\right)^{2}} e^{-\beta u_{\mathbf{p} \mathbf{0}}})\right|_{G=0} \\
& =\left.\frac{p_{0}^{2}}{k T}\left(\frac{1}{Z} \sum_{\mathbf{p}_{\mathbf{0}}} \cos ^{2} \theta e^{-\beta u_{\mathbf{p}_{\mathbf{0}}}}\right)\right|_{G=0} \\
& =\left.\frac{p_{0}^{2}}{k T}\left(\overline{\cos ^{2} \theta}\right)\right|_{G=0} .
\end{aligned}
$$

Só falta, então, calcularmos o valor médio de $\cos ^{2} \theta$, avaliado para $G=0$ - o que simplifica o cálculo, porque com $G=0$ temos $e^{-\beta u_{\mathbf{p}_{\mathbf{0}}}}=1$, e, assim, trata-se de uma média simples. Em coordenadas esféricas (trocando o somatório em $\mathbf{p}_{\mathbf{0}}$ por integrais), temos

$$
\left.\left(\overline{\cos ^{2} \theta}\right)\right|_{G=0}=\frac{\int_{0}^{2 \pi} \int_{0}^{\pi} \cos ^{2} \theta \operatorname{sen} \theta \mathrm{d} \theta \mathrm{d} \phi}{\int_{0}^{2 \pi} \int_{0}^{\pi} \operatorname{sen} \theta \mathrm{d} \theta \mathrm{d} \phi}=\frac{1}{3},
$$

9 Um ponto como este não seria tão simples se estivéssemos considerando polarizabilidades moleculares não-nulas (ou não desprezíveis), percebe? 
como você pode facilmente verificar, e então

$$
\left.\frac{\partial\left(\overline{\mathbf{p}_{\mathbf{0}}} \cdot \hat{\mathbf{z}}\right)}{\partial G}\right|_{G=0}=\frac{p_{0}^{2}}{3 k T} .
$$

Substituindo (21) em (16), obtemos finalmente a igualdade 13 .

\section{Obtendo a Equação de Kirkwood}

Sabemos que para o cálculo do campo interno $\left(\mathbf{E}_{\mathbf{i n t}}\right)$ do modelo de Onsager a molécula considerada é imaginada em uma cavidade esférica microscópica, e além de tal cavidade o fluido dielétrico é modelado como um meio contínuo (veja a Fig. 1). Como dissemos em nosso artigo anterior [2], rigorosamente isso não faz sentido - especialmente se considerarmos as moléculas mais próximas da molécula na cavidade imaginária. Kirkwood procurou melhorar essa modelagem [3, ao substituir a cavidade microscópica da Fig. 1] por uma "cavidade macroscópica", ilustrada aqui na Fig. 2 O termo "cavidade", neste caso, talvez não seja o mais adequado. Podemos, simplesmente, dizer que no modelo de Kirkwood as moléculas do fluido dielétrico são tratadas de forma discreta no interior da uma pequena esfera macroscópica, de raio $a_{\text {macro, }}$ e além dessa esfera o fluido é modelado como um meio contínuo. Por outro lado, uma limitação do modelo de Kirkwood (posteriormente contornada por Fröhlich) é que seu desenvolvimento se aplica apenas a moléculas não polarizáveis (ou com polarizabilidades desprezíveis), e, assim, cada molécula no interior da esfera macroscópica da Fig. 2 é inicialmente descrita apenas como um momento de dipolo permanente, de módulo $p_{0}$.

Seja M o momento de dipolo total da porção do fluido na esfera ilustrada na Fig. 2 , de volume $\mathcal{V}$. Considerando o fluido submetido a um campo externo que resulta em um campo macroscópico uniforme $\mathbf{E}$ no mesmo, e também que o meio é linear, podemos expressar sua polarização elétrica comø 10

$$
\mathbf{P}=\frac{\overline{\mathbf{M}}}{\mathcal{V}}
$$

e adaptar os Passos $1^{\prime}$ e $2^{\prime}$ da seção anterior (agora visando a obtenção da equação de Kirkwood) para:

- Passo 1": Usando mecânica estatística, calcule $\overline{\mathbf{M}}$ para o fluido na esfera macroscópica da Fig. 2, e então obtenha

$$
\mathbf{P}=\frac{\overline{\mathbf{M}}}{\mathcal{V}}=N \frac{\overline{\mathbf{M}}}{N \mathcal{V}}=N \frac{\overline{\mathbf{M}}}{\mathcal{N}},
$$

\footnotetext{
10 Tratando-se de uma porção macroscópica de um fluido homogêneo submetido a um campo elétrico uniforme e constante no tempo, $\mathbf{M}$ é constante - a menos de flutuações estatísticas ínfimas. Assim, na igualdade $\mathbf{P}=\overline{\mathbf{M}} / \mathcal{V}$ poderíamos escrever $\mathbf{M}$, em vez de $\overline{\mathbf{M}}$, como frequentemente é o caso para certas grandezas termodinâmicas (pressão, por exemplo). Mas porque no desenvolvimento do modelo de Kirkwood são realizadas manipulações relativas ao cálculo da média de $\mathbf{M}$, é necessário trabalharmos com $\overline{\mathbf{M}}$, em vez de $\mathbf{M}$.
}

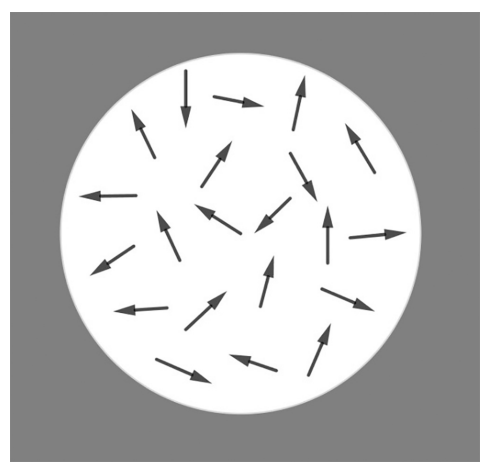

Figura 2: Esfera macroscópica imaginária relativa ao modelo de Kirkwood para fluidos polares puros. As $\mathcal{N}$ moléculas dentro da esfera são aqui apresentadas como dipolos permanentes (explicitados, nesta figura, em número bastante reduzido, é claro), e fora dela o dielétrico é modelado como um meio contínuo.

em que $N$ é o número de moléculas por unidade de volume do fluido e $\mathcal{N}$ é o número de moléculas na esfera macroscópica.

- Passo 2 ${ }^{\prime \prime}$ : Substitua o módulo de $\mathbf{P}$ (que, supõe-se, é proporcional a $E$ ) na igualdade

$$
\epsilon_{r}=1+\frac{P}{\epsilon_{0} E} .
$$

Como antes, podemos reunir esses dois passos em um só:

- Usando mecânica estatística, calcule $\overline{\mathbf{M}}$ para o fluido na esfera macroscópica da Fig. 2, e substitua a expressão obtida na igualdade

$$
\epsilon_{0}\left(\epsilon_{r}-1\right) E=\frac{N}{\mathcal{N}} \overline{\mathbf{M}} \cdot \hat{\mathbf{z}},
$$

em que $\hat{\mathbf{z}}$ é o versor com a direção e o sentido do campo macroscópico $\mathbf{E}$.

Perceba que a igualdade 22 recai na igualdade (4) no caso particular em que $\mathcal{N}^{N}=1$ (e sempre que fizermos $\mathcal{N}=1$, estaremos fazendo também $a_{\text {macro }}=a$, ou seja, estaremos reduzindo a esfera macroscópica da Fig. 2 à cavidade microscópica da Fig. 1).

Agora, seguiremos um desenvolvimento muito semelhante ao desenvolvimento alternativo apresentado na seção anterior - evitando, assim, a tentativa de um cálculo explícito de $\overline{\mathbf{M}}$. Destacaremos em que pontos aquele desenvolvimento corresponde a um caso particular do que veremos a seguir, e em que pontos há diferenças cruciais. Com isso, acreditamos que estaremos contribuindo não apenas para deixar mais clara a relação entre os modelos de Onsager e Kirkwood, mas também para uma melhor compreensão do próprio modelo de Kirkwood.

Expandindo $\overline{\mathbf{M}} \cdot \hat{\mathbf{z}}$ (lembrando que $\hat{\mathbf{z}}$ é o versor com a direção e o sentido do campo $\mathbf{E}$ ) em potências de $E$ $(E=\mathbf{E} \cdot \hat{\mathbf{z}})$ de expoentes não negativos, a partir de 
$E=0$, e tomando apenas os dois primeiros termos (considerando que a intensidade $E$ do campo macroscópico E é suficientemente baixa), obtemos

$$
(\overline{\mathbf{M}} \cdot \hat{\mathbf{z}})(E) \approx(\overline{\mathbf{M}} \cdot \hat{\mathbf{z}})(0)+\left.\frac{\partial(\overline{\mathbf{M}} \cdot \hat{\mathbf{z}})}{\partial E}\right|_{E=0} E .
$$

Mas para $E=0$ temos $\overline{\mathbf{M}}=0$, e, portanto (a partir daqui usando "=", em vez de “ $\approx ")$,

$$
\overline{\mathbf{M}} \cdot \hat{\mathbf{z}}=\left.\frac{\partial(\overline{\mathbf{M}} \cdot \hat{\mathbf{z}})}{\partial E}\right|_{E=0} E .
$$

É claro, a igualdade 14 é um caso particular da igualdade (23), para $\mathcal{N}=1$.

O próximo passo consiste em fazermos, no segundo membro da igualdade 23 - mais especificamente em $\left.[\partial(\overline{\mathbf{M}} \cdot \hat{\mathbf{z}}) / \partial E]\right|_{E=0}{ }^{-}$, uma mudança de variável.

A motivação para a realização de uma mudança de variável em $\left.[\partial(\overline{\mathbf{M}} \cdot \hat{\mathbf{z}}) / \partial E]\right|_{E=0}$ é que o cálculo de $\overline{\mathbf{M}}$ não envolve diretamente o campo $\mathbf{E}$, mas o campo $\mathbf{E}_{\text {macro }}$ que age sobre as moléculas na esfera macroscópica da Fig. 2. Sendo $\mathbf{E}_{\text {macro }}$ uma função de $\mathbf{E}$, com $\mathbf{E}_{\text {macro }}=0$ para $\mathbf{E}=0$, podemos reescrever a igualdade 23 como

$$
\overline{\mathbf{M}} \cdot \hat{\mathbf{z}}=\left.\left.\frac{\partial(\overline{\mathbf{M}} \cdot \hat{\mathbf{z}})}{\partial E_{\text {macro }}}\right|_{E_{\text {macro }}=0} \frac{\partial E_{\text {macro }}}{\partial E}\right|_{E=0} E,
$$

e combinando-a com a igualdade 22 obtemos

$$
\epsilon_{0}\left(\epsilon_{r}-1\right)=\left.\left.\frac{N}{\mathcal{N}} \frac{\partial(\overline{\mathbf{M}} \cdot \hat{\mathbf{z}})}{\partial E_{\text {macro }}}\right|_{E_{\text {macro }}=0} \frac{\partial E_{\text {macro }}}{\partial E}\right|_{E=0} .
$$

Kirkwood [3] considerou $\mathbf{E}_{\text {macro }}=\mathbf{G}$, que é o campo de cavidade gerado pelo meio contínuo da Fig.2 Assim, temos, no modelo de Kirkwood:

$$
\mathbf{E}_{\text {macro }}=\mathbf{G}=\left(\frac{3 \epsilon_{r}}{2 \epsilon_{r}+1}\right) \mathbf{E}
$$

Pois bem, substituindo 25 em 24) obtemos

$$
\begin{aligned}
\frac{\left(\epsilon_{r}-1\right)\left(2 \epsilon_{r}+1\right)}{9 \epsilon_{r}} & =\left.\frac{N}{3 \epsilon_{0}} \frac{1}{\mathcal{N}} \frac{\partial(\overline{\mathbf{M}} \cdot \hat{\mathbf{z}})}{\partial E_{\text {macro }}}\right|_{E_{\text {macro }}=0} \\
& =\left.\frac{N}{3 \epsilon_{0}} \frac{1}{\mathcal{N}} \frac{\partial(\overline{\mathbf{M}} \cdot \hat{\mathbf{z}})}{\partial G}\right|_{G=0} .
\end{aligned}
$$

Agora, vamos trabalhar a quantidade

$$
\left.\frac{\partial(\overline{\mathbf{M}} \cdot \hat{\mathbf{z}})}{\partial E_{\text {macro }}}\right|_{E_{\text {macro }}=0}=\left.\frac{\partial(\overline{\mathbf{M}} \cdot \hat{\mathbf{z}})}{\partial G}\right|_{G=0},
$$

em (26), de modo semelhante a como trabalhamos, na seção anterior, a quantidade $\left.\left[\partial\left(\overline{\mathbf{p}_{\mathbf{0}}} \cdot \hat{\mathbf{z}}\right) / \partial G\right]\right|_{G=0}$.

O primeiro passo consiste em obtermos uma expressão para $\overline{\mathbf{M}}$ - sem, contudo, termos a pretensão de realizar um cálculo explícito dessa média. Trabalhando com o ensemble canônico da mecânica estatística, considerando o meio dielétrico além da esfera macroscópica da Fig. 2 como o reservatório térmico, podemos escrever (compare com a igualdade (5):

$$
\overline{\mathbf{M}}=\frac{\sum_{r} \mathbf{M}_{r} e^{-\beta \varepsilon_{r}}}{\sum_{r} e^{-\beta \varepsilon_{r}}},
$$

em que $\varepsilon_{r}$ é a energia de um determinado microestado $r$ do sistema de dipolos permanentes da Fig. 2, e os somatórios são realizados sobre todos os microestados acessíveis àquele sistema. Um desenvolvimento semelhante ao apresentado entre as igualdades (5) e (7) nos leva a

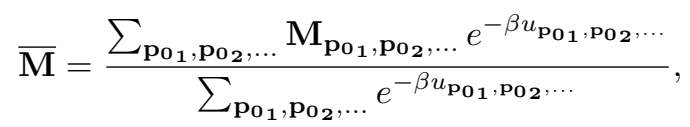

em que a sequência $\mathbf{p}_{0_{1}}, \mathbf{p}_{\mathbf{0}_{2}}, \ldots$ - que é uma abreviação para $\mathbf{p}_{\mathbf{0}_{1}}, \mathbf{p}_{\mathbf{0}_{2}}, \ldots, \mathbf{p}_{\mathbf{0}_{\mathcal{N}}}$ - indica um determinado microestado do sistema de dipolos permanentes da Fig. 2 Estamos considerando, no modelo, os $\mathcal{N}$ dipolos em posições fixas (apenas livres para rotacionar), mas, alternativamente, podemos dar a cada um desses dipolos 3 graus de liberdade adicionais, permitindo-os transladar pela região esférica macroscópica ilustrada na Fig. 2 Neste caso, a sequência $\mathbf{p}_{\mathbf{0}_{1}}, \mathbf{p}_{\mathbf{0}_{\mathbf{2}}}, \ldots, \mathbf{p}_{\mathbf{0} \mathcal{N}}$, que indica um determinado microestado do sistema de dipolos permanentes da Fig. 2, deve ser substituída pela sequência $\left(\mathbf{r}_{\mathbf{1}}, \mathbf{p}_{\mathbf{0}_{1}}\right),\left(\mathbf{r}_{\mathbf{2}}, \mathbf{p}_{\mathbf{0}_{\mathbf{2}}}\right), \ldots,\left(\mathbf{r}_{\mathcal{N}}, \mathbf{p}_{\mathbf{0}_{\mathcal{N}}}\right)$, em que $\mathbf{r}_{\mathbf{i}}$ é o vetor posição do $i$-ésimo dipolo. Mas é possível mostrar que tal substituição não levaria a qualquer mudança fundamental nos resultados que serão obtidos nesta e na próxima seção. Continuando, podemos expressar $u_{\mathbf{p}_{\mathbf{0}_{1}}, \mathbf{p}_{\mathbf{0}_{2}}, \ldots}$ como a soma de dois grandes termos: a energia $u_{\text {dip-dip }}$ de interação entre os dipolos permanentes na esfera macroscópica da Fig. 2, e a energia $u_{\text {dip }}-\mathbf{E}_{\text {macro }}$ de interação desses dipolos com o campo $\mathbf{E}_{\text {macro }}$ - lembrando que, no modelo de Kirkwood, $\mathbf{E}_{\text {macro }}=\mathbf{G}$. Assim, temos:

$$
u_{\mathbf{p}_{\mathbf{0}_{1}}, \mathbf{p}_{\mathbf{2}}, \ldots}=u_{\mathrm{dip}-\operatorname{dip}}+u_{\mathrm{dip}-\mathbf{E}_{\mathrm{macro}}}
$$

com (observando que $\mathbf{E}_{\text {macro }}$ é um campo uniforme)

$$
\begin{aligned}
u_{\text {dip }-\mathbf{E}_{\text {macro }}} & =-\mathbf{p}_{\mathbf{0}_{\mathbf{1}}} \cdot \mathbf{E}_{\text {macro }}-\mathbf{p}_{\mathbf{0}_{\mathbf{2}}} \cdot \mathbf{E}_{\text {macro }}+\cdots \\
& =-\left(\mathbf{p}_{\mathbf{0}_{1}}+\mathbf{p}_{\mathbf{0}_{2}}+\cdots\right) \cdot \mathbf{E}_{\text {macro }}+\cdots \\
& =-\mathbf{M}_{\mathbf{p}_{\mathbf{0}_{1}}, \mathbf{p}_{\mathbf{0}_{2}}, \ldots} \cdot \mathbf{E}_{\text {macro }} \\
& =-\mathbf{M}_{\mathbf{p}_{\mathbf{0}_{1}}, \mathbf{p}_{\mathbf{2}}, \ldots}, E_{\text {macro }} \hat{\mathbf{z}} \\
& =-\left(\mathbf{M}_{\mathbf{p}_{\mathbf{0}_{1}}, \mathbf{p}_{\mathbf{2}}, \ldots} \cdot \hat{\mathbf{z}}\right) E_{\text {macro }} .
\end{aligned}
$$

Portanto,

$$
u_{\mathbf{p}_{\mathbf{0}_{1}}, \mathbf{p}_{\mathbf{0}_{2}}, \ldots}=u_{\mathrm{dip}-\operatorname{dip}}-\left(\mathbf{M}_{\mathbf{p}_{\mathbf{0}_{1}}, \mathbf{p}_{\mathbf{0}_{2}}, \ldots} \cdot \hat{\mathbf{z}}\right) E_{\text {macro }} .
$$

Tomando o produto escalar com $\hat{\mathbf{z}}$ de ambos os membros da igualdade (27), obtemos:

$$
\overline{\mathbf{M}} \cdot \hat{\mathbf{z}}=\frac{1}{Z} \sum_{\mathbf{p}_{\mathbf{0}_{1}}, \mathbf{p}_{\mathbf{0}_{2}}, \ldots}\left(\mathbf{M}_{\mathbf{p}_{\mathbf{o}_{1}}, \mathbf{p}_{\mathbf{o}_{2}}, \ldots} \cdot \hat{\mathbf{z}}\right) e^{-\beta u_{\mathbf{p}_{\mathbf{O}_{1}}, \mathbf{p}_{\mathbf{2}}, \cdots}, \ldots}
$$


com

$$
Z=\sum_{\mathbf{p}_{\mathbf{0}_{1}, \mathbf{p}_{\mathbf{2}}, \ldots}} e^{-\beta u_{\mathbf{p}_{\mathbf{0}}, \mathbf{p}_{\mathbf{0}}, \ldots}, \ldots} .
$$

As igualdades (30) e 31, com a expressão para $u_{\mathbf{p}_{0_{1}}, \mathbf{p}_{\mathbf{0}_{2}}, \ldots}$ em $(29)$, e que são válidas para $\mathcal{N} \ggg 1$, correspondem às igualdades (17) e (18) (nas quais temos $\mathcal{N}=1)$.

Um desenvolvimento muito semelhante ao que nos levou, na seção anterior, da igualdade (17) à igualdade 20, nos leva da igualdade (30) a

$$
\begin{aligned}
& \left.\frac{\partial(\overline{\mathbf{M}} \cdot \hat{\mathbf{z}})}{\partial E_{\text {macro }}}\right|_{E_{\text {macro }}=0} \\
& =-\left.\frac{\beta}{Z} \sum_{\mathbf{p o}_{\mathbf{1}_{1}}, \ldots}\left(\mathbf{M}_{\mathbf{p}_{\mathbf{0}_{1}}, \ldots} \cdot \hat{\mathbf{z}}\right) \frac{\partial u_{\mathbf{p}_{\mathbf{0}_{1}}, \ldots}}{\partial E_{\text {macro }}} e^{-\beta u_{\mathbf{p}_{\mathbf{1}}, \ldots}}\right|_{E_{\text {macro }}=0} .
\end{aligned}
$$

Ao realizar esse desenvolvimento, observe, particularmente, que

$$
\left.(\overline{\mathbf{M} \cdot \hat{\mathbf{z}}})\right|_{E_{\text {macro }}=0}=\left.(\overline{\mathbf{M}} \cdot \hat{\mathbf{z}})\right|_{E=0}=\overbrace{\left(\left.\overline{\mathbf{M}}\right|_{E=0}\right)}^{0 \hat{\mathbf{z}}} \cdot \hat{\mathbf{z}}=0,
$$

e que

$$
\begin{gathered}
\frac{\partial}{\partial E_{\text {macro }}}\left(\mathbf{M}_{\mathbf{p}_{\mathbf{0}_{1}}, \mathbf{p}_{\mathbf{2}}, \ldots} \cdot \hat{\mathbf{z}}\right)=\left(\frac{\partial \mathbf{M}_{\mathbf{p}_{\mathbf{o}_{1}}, \mathbf{p}_{\mathbf{2}}, \ldots}, \ldots}{\partial E_{\text {macro }}}\right) \cdot \hat{\mathbf{z}} \\
=\left(\frac{\partial \mathbf{p}_{\mathbf{0}_{\mathbf{1}}}}{\partial E_{\text {macro }}}+\frac{\partial \mathbf{p}_{\mathbf{0}_{\mathbf{2}}}}{\partial E_{\text {macro }}}+\cdots\right) \cdot \hat{\mathbf{z}}=0
\end{gathered}
$$

pois $\mathbf{p}_{\mathbf{0}_{1}}, \mathbf{p}_{\mathbf{0}_{2}}, \ldots$ não são funções de $E_{\text {macro }}{ }^{11}$

Substituindo 29 em 32 obtemos (e a partir daqui você observará diferenças interessantes em relação ao desenvolvimento realizado no final da seção anterior, para o caso $\mathcal{N}=1$ ):

$$
\begin{aligned}
& \left.\frac{\partial(\overline{\mathbf{M}} \cdot \hat{\mathbf{z}})}{\partial E_{\text {macro }}}\right|_{E_{\text {macro }}=0} \\
& \quad=\left.\beta\left(\frac{1}{Z} \sum_{\mathbf{p}_{\mathbf{0}_{1}}, \ldots}\left(\mathbf{M}_{\mathbf{p}_{\mathbf{0}_{\mathbf{1}}, \ldots}, \ldots} \cdot \hat{\mathbf{z}}\right)^{2} e^{-\beta u_{\mathbf{p}_{\mathbf{0} 1}, \ldots}}\right)\right|_{E_{\text {macro }}=0} \\
& \quad=\left.\beta \frac{(\mathbf{M} \cdot \hat{\mathbf{z}})^{2}}{}\right|_{E_{\text {macro }}=0} .
\end{aligned}
$$

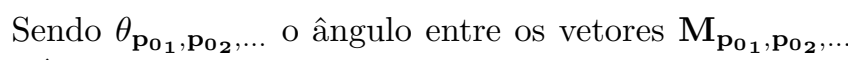
e $\hat{\mathbf{z}}$, temos

$$
\mathbf{M}_{\mathbf{p}_{\mathbf{o}_{1}}, \mathbf{p}_{\mathbf{2}}, \ldots} \cdot \hat{\mathbf{z}}=\left(M_{\mathbf{p}_{\mathbf{o}_{1}}, \mathbf{p}_{\mathbf{o}_{2}}, \ldots}\right) \cos \left(\theta_{\mathbf{p}_{\mathbf{0}_{1}}, \mathbf{p}_{\mathbf{2}}, \ldots}\right),
$$

e podemos então reescrever a igualdade 33 como

$$
\begin{aligned}
& \left.\frac{\partial(\overline{\mathbf{M}} \cdot \hat{\mathbf{z}})}{\partial E_{\text {macro }}}\right|_{E_{\text {macro }}=0}=\left.\beta \overline{M^{2} \cos ^{2} \theta}\right|_{E_{\text {macro }}=0} \\
& \quad=\beta \overline{\left(\left.M^{2}\right|_{E_{\text {macro }}=0}\right)\left(\left.\cos ^{2} \theta\right|_{E_{\text {macro }}=0}\right) .}
\end{aligned}
$$

\footnotetext{
11 Novamente, um ponto como este não seria tão simples se estivéssemos considerando polarizabilidades moleculares não-nulas (ou não desprezíveis).
}

Agora, perceba que as variáveis $\left.M^{2}\right|_{E_{\text {macro }}=0} \mathrm{e}$ $\left.\cos ^{2} \theta\right|_{E_{\text {macro }=0}}$ são estatisticamente independentes ${ }^{12} \mathrm{e}$ assim a média de seu produto é igual ao produto de suas médias, e portanto podemos reescrever a igualdade (34) como

$$
\left.\frac{\partial(\overline{\mathbf{M}} \cdot \hat{\mathbf{z}})}{\partial E_{\text {macro }}}\right|_{E_{\text {macro }}=0}=\beta\left(\overline{\left.M^{2}\right|_{E_{\text {macro }}=0}}\right)\left(\overline{\left.\cos ^{2} \theta\right|_{E_{\text {macro }}=0}}\right) .
$$

Porque não há, com $\mathbf{E}_{\text {macro }}=0$, orientação preferencial para $\mathbf{M}$, o cálculo desta última média é idêntico ao realizado para $\left.\left(\overline{\cos ^{2} \theta}\right)\right|_{G=0}$ no final da seção anterior. Temos, então,

$$
\overline{\left.\cos ^{2} \theta\right|_{E_{\text {macro }}=0}}=\frac{1}{3},
$$

e com isso obtemos

$$
\left.\frac{\partial(\overline{\mathbf{M}} \cdot \hat{\mathbf{z}})}{\partial E_{\text {macro }}}\right|_{E_{\text {macro }}=0}=\frac{1}{3 k T} \overline{\left(M^{2}\right)_{0}},
$$

em que usamos a abreviação $\overline{\left(M^{2}\right)_{0}}$ para $\overline{\left.M^{2}\right|_{E_{\text {macro }}=0}}$. No caso $\mathcal{N}=1$ temos, no lugar de $\overline{\left(M^{2}\right)_{0}}, p_{0}^{2}$, e a igualdade (35) recai na igualdade 21).

Vamos trabalhar a média no segundo membro da igualdade (35). Temos:

$$
\begin{aligned}
\overline{\left(M^{2}\right)_{0}} & =\overline{(\mathbf{M} \cdot \mathbf{M})_{0}}=\overline{\left[\left(\sum_{i=1}^{\mathcal{N}} \mathbf{p}_{\mathbf{0}_{\mathbf{i}}}\right) \cdot\left(\sum_{j=1}^{\mathcal{N}} \mathbf{p}_{\mathbf{0}_{\mathbf{j}}}\right)\right]_{0}} \\
& =\overline{(\sum_{i=1}^{\mathcal{N}} \sum_{j=1}^{\mathcal{N}} \overbrace{\mathbf{p}_{0}^{2} \cos \theta_{i j}}^{\mathbf{p}_{\mathbf{0}_{\mathbf{j}}}})_{0}}=p_{0}^{2} \sum_{i=1}^{\mathcal{N}} \sum_{j=1}^{\mathcal{N}} \overline{\left(\cos \theta_{i j}\right)_{0}},
\end{aligned}
$$

em que $\theta_{i j}$ é o ângulo entre os vetores $\mathbf{p}_{\mathbf{0}_{\mathbf{i}}}$ e $\mathbf{p}_{\mathbf{0}_{\mathbf{j}}}$, em um determinado sistema do ensemble (ou em um determinado instante, considerando-se a hipótese ergódica). Assim, para $i \neq j, \overline{\left(\cos \theta_{i j}\right)_{0}}$ é a média, no ensemble ${ }^{13}$ do cosseno do ângulo entre dois dipolos $i$ e $j$ na esfera macroscópica da Fig. 2, com $E_{\text {macro }}=0$ (ou seja, com o capacitor descarregado). E para $i=j$ temos, é claro, $\overline{\left(\cos \theta_{i j}\right)_{0}}=1$. Definindo

$$
g \equiv \frac{1}{\mathcal{N}} \sum_{i=1}^{\mathcal{N}} \sum_{j=1}^{\mathcal{N}} \overline{\left(\cos \theta_{i j}\right)_{0}}
$$

que é o chamado fator de correlação de Kirkwood (ou fator $g$ de Kirkwood), podemos reescrever:

$$
\overline{\left(M^{2}\right)_{0}}=\mathcal{N} p_{0}^{2} g
$$

\footnotetext{
$\overline{12} \mathrm{Na}$ ausência do campo $\mathbf{E}_{\text {macro, não há orientação preferencial }}$ para o vetor $\mathbf{M}$.

13 Lembre-se que estamos trabalhando com o ensemble canônico, considerando como o reservatório térmico o meio dielétrico além da esfera macroscópica da Fig. 2 .
} 
e com isso a igualdade 35 fica $\sqrt{14}$

$$
\left.\frac{\partial(\overline{\mathbf{M}} \cdot \hat{\mathbf{z}})}{\partial E_{\text {macro }}}\right|_{E_{\text {macro }}=0}=\mathcal{N} \frac{p_{0}^{2}}{3 k T} g .
$$

Substituindo $(37)$ em 26 obtemos ${ }^{15}$

$$
\frac{\left(\epsilon_{r}-1\right)\left(2 \epsilon_{r}+1\right)}{9 \epsilon_{r}}=\frac{N}{3 \epsilon_{0}} \frac{p_{0}^{2}}{3 k T} g,
$$

que é a equação de Kirkwood para fluidos polares puros de moléculas não polarizáveis (ou com polarizabilidades desprezíveis).

\section{Sobre o Fator de Correlação de Kirkwood $(g)$}

O fator de correlação de Kirkwood, definido através da relação (36), pode ser pensado como uma expressão da correlação entre as orientações das moléculas do fluido, na situação de campo elétrico externo nulo.

$\mathrm{Na}$ ausência de correlação, temos $\overline{\left(\cos \theta_{i j}\right)_{0}}=\delta_{i j}-$ ou seja, $\overline{\left(\cos \theta_{i j}\right)_{0}}=0$, se $i \neq j$, e $\overline{\left(\cos \theta_{i j}\right)_{0}}=1$, se $i=j$ (tratando-se, neste caso, de um mesmo dipolo), e assim obtemos:

$$
g=\frac{1}{\mathcal{N}} \sum_{i=1}^{\mathcal{N}} \sum_{j=1}^{\mathcal{N}} \delta_{i j}=\frac{1}{\mathcal{N}} \sum_{i=1}^{\mathcal{N}} 1=\frac{\mathcal{N}}{\mathcal{N}}=1 .
$$

Motivados por este resultado, e observando que com ou sem correlação temos $\overline{\left(\cos \theta_{i i}\right)_{0}}=1$ (já que se trata de um mesmo dipolo), podemos escrever, a partir de (36):

$$
\begin{aligned}
g & =\frac{1}{\mathcal{N}} \sum_{i=1}^{\mathcal{N}}\left(\overline{\left(\cos \theta_{i i}\right)_{0}}+\sum_{j \neq i}^{\mathcal{N}} \overline{\left(\cos \theta_{i j}\right)_{0}}\right) \\
& =1+\frac{1}{\mathcal{N}} \sum_{i=1}^{\mathcal{N}} \sum_{j \neq i}^{\mathcal{N}} \overline{\left(\cos \theta_{i j}\right)_{0}} .
\end{aligned}
$$

Se $\overline{\left(\cos \theta_{i j}\right)_{0}}>0(\operatorname{com} i \neq j)$, temos $g>1$.

\footnotetext{
14 Não resistimos citar, aqui, o seguinte trecho do livro Física Moderna para iniciados, interessados e aficionados 5, de Ivan S. Oliveira, pesquisador titular do Centro Brasileiro de Pesquisas Físicas: "Em física é assim: tratamos com pompa e detalhes matemáticos o que conhecemos; ao que não conhecemos associamos uma letra qualquer, damos um nome pomposo, e incluímos nos cálculos. E bárbaro!" O autor estava se referindo (na página 144 do volume 1) ao conceito de massa efetiva, mas a citação reflete bem o espírito da introdução do fator de correlação de Kirkwood no desenvolvimento de seu modelo, já que seu cálculo deve ser feito à parte, e pode ser bastante desafiador. Mas, frequentemente, é assim que os físicos trabalham, quando desenvolvem modelos. Discutiremos o fator $g$ na próxima seção.

15 Perceba que todo o desenvolvimento que realizamos após a igualdade 26 não envolve a expressão para $E_{\text {macro. A única }}$ exigência feita, após a igualdade (26), foi $\mathbf{E}_{\text {macro }}$ ser um campo uniforme.
}

O valor de

$$
\sum_{j \neq i}^{\mathcal{N}} \overline{\left(\cos \theta_{i j}\right)_{0}}
$$

em (40), depende do dipolo $i$ considerado? Em uma primeira análise, podemos imaginar que sim, porque deve fazer diferença se o dipolo $i$ está, por exemplo, próximo à borda da esfera da Fig. 2 ou próximo ao seu centro. Nestes dois casos, o dipolo $i$ "enxerga" de formas distintas os demais dipolos na esfera macroscópica. Contudo, se, na situação de campo elétrico externo nulo, é desprezível a correlação entre as orientações de moléculas do fluido distantes entre si por alguns diâmetros moleculares (como é típico para líquidos), e lembrando que o valor de $\mathcal{N}$ é bastante elevado (já que é macroscópica a esfera da Fig. 2), então o valor de $\sum_{j \neq i}^{\mathcal{N}} \overline{\left(\cos \theta_{i j}\right)_{0}}$ não depende (com ótima aproximação) de $i$, e pode ser reescrito como $\sum_{j \neq i}^{\tilde{\mathcal{N}}} \overline{\left(\cos \theta_{i j}\right)_{0}}$, sendo $\tilde{\mathcal{N}}$ o número de dipolos $j$ vizinhos ao dipolo $i$ tais que $\overline{\left(\cos \theta_{i j}\right)_{0}} \neq 0$. Para líquidos típicos temos, deve estar claro, $\tilde{\mathcal{N}} \lll \mathcal{N}$. Com essas considerações obtemos, a partir de 40 :

$$
g \approx 1+\frac{1}{\mathcal{N}}\left(\mathcal{N} \sum_{j \neq i}^{\tilde{\mathcal{N}}} \overline{\left(\cos \theta_{i j}\right)_{0}}\right)=1+\sum_{j \neq i}^{\tilde{\mathcal{N}}} \overline{\left(\cos \theta_{i j}\right)_{0}}
$$

em que $i$ é um dipolo qualquer na esfera macroscópica da Fig. 2 (e, por isso mesmo, pode ser pensado como o dipolo no centro daquela esfera), correlacionado, em termos de orientação, com apenas $\tilde{\mathcal{N}}$ dipolos vizinhos.

É interessante observar que podemos reescrever a relação (36) como

$$
g=\frac{1}{\mathcal{N}} \sum_{i=1}^{\mathcal{N}} g_{i}=\overline{g_{i}}
$$

com

$$
g_{i} \equiv \sum_{j=1}^{\mathcal{N}} \overline{\left(\cos \theta_{i j}\right)_{0}} .
$$

Se $g_{i}$ é aproximadamente independente de $i$, temos $\overline{g_{i}} \approx$ $g_{i}$, e, portanto,

$$
g \approx \sum_{j=1}^{\mathcal{N}} \overline{\left(\cos \theta_{i j}\right)_{0}}
$$

o que reproduz $(\operatorname{com} \mathcal{N}$ substituído por $\tilde{\mathcal{N}}$, e com o termo $\overline{\left(\cos \theta_{i i}\right)_{0}}=1$ explicitado) a aproximação em 41 .

Se a correlação entre as orientações das moléculas do fluido (na situação de campo elétrico externo nulo) já é desprezível além de suas z "primeiras vizinhas", e se $\overline{\left(\cos \theta_{i j}\right)_{0}}$ independe de qual é a primeira vizinha considerada, obtemos (como fez Kirkwood em seu artigo [3]) uma aproximação ainda mais simples para $g$ (veja (41)):

$$
g \approx 1+z \overline{\left(\cos \theta_{i j}\right)_{0}},
$$


em que $i$ e $j$ denotam duas moléculas que são primeiras vizinhas.

Em 1933, Bernal e Fowler, da Universidade de Cambridge, publicaram um artigo intitulado "A theory of water and ionic solution, with particular reference to hydrogen and hydroxyl ions" [6], no qual propõem um modelo para a molécula de água (a partir de dados espectroscópicos) e para a estrutura interna da água líquida, e então deduzem várias propriedades para este líquido e para soluções iônicas. Kirkwood 3 extraiu daquele trabalho (para água líquida a temperaturas acima de 4 graus Celsius) $z=4$ (lembrando que $z$, em 42 , é o número de primeiras vizinhas de uma molécula de água, no estado líquidd ${ }^{16}$ e, indiretamente, $\overline{\left(\cos \theta_{i j}\right)_{0}}=0,41$, e com isso obteve (usando a aproximação em (42)) $g \approx 2,64$

Um cálculo de $g$ bem mais recente, e muito mais complexo, para água líquida foi realizado por Zhang, Hutter e Sprik [7] (Zhang e Sprik também da Universidade de Cambridge, e Hutter da Universidade de Zurique). Eles fizeram uso de métodos computacionais avançados, envolvendo a chamada teoria do funcional da densidade (uma teoria quântica muito usada na investigação da estrutura eletrônica ou nuclear - principalmente do estado fundamental - de sistemas de muitos corpos, em particular átomos, moléculas e fases condensadas). Obtiveram $g \approx 2,2-$ um valor não tão diferente daquele obtido por Kirkwood 77 anos antes ${ }^{18}$

O momento de dipolo permanente de uma molécula de água (isolada) tem módulo $p_{0} \approx 6,14 \times 10^{-30} \mathrm{C} \cdot \mathrm{m}$, e para água pura a $1 \mathrm{~atm}$ e $20^{\circ} \mathrm{C}(T=293 \mathrm{~K})$ temos $N \approx 3,34 \times 10^{28} \mathrm{~m}^{-3}$. Com estes valores, a quantidade adimensional no segundo membro de $(38)$ fica:

$$
\frac{N}{3 \epsilon_{0}} \frac{p_{0}^{2}}{3 k T} g \approx 10,3, \quad \text { para } g=2,64,
$$

$$
\frac{N}{3 \epsilon_{0}} \frac{p_{0}^{2}}{3 k T} g \approx 8,60, \quad \text { para } g=2,20 \text {. }
$$

Substituindo os dois valores acima no segundo membro da igualdade (38), e resolvendo a equação resultante para $\epsilon_{r}$, obtemos, respectivamente, $\epsilon_{r} \approx 46,9$ (para

\footnotetext{
16 Esse número é denominado "número de coordenação".

17 Como curiosidade, saiba que Kirkwood não fez uso, em seu artigo [3, da letra " $g$ " ou de qualquer outra letra para representar a quantidade à qual ela se refere. Mas hoje esta é a letra usada por todos os pesquisadores, quando se referem àquela quantidade. Não à toa o fator de correlação de Kirkwood $(g)$ é também denominado "fator $g$ de Kirkwood".

18 Consideramos interessante frisar, para os estudantes menos experientes, que o cálculo de $g$ para a água realizado por Zhang e colaboradores 7, e mesmo o cálculo de $g$ realizado por Kirkwood [3], e partir do trabalho de Bernal e Fowler 6], não se limita ao uso da eletrodinâmica clássica e da mecânica estatística clássica (que foram suficientes para a obtenção da equação de Kirkwood). Isso é muito interessante, e contribui para o entendimento de como a física "funciona", de como os físicos trabalham.
}

$g=2,64)$ e $\epsilon_{r} \approx 39,2$ (para $g=2,20$ ). São valores ainda distantes do valor experimental para a constante dielétrica da água $\left(\epsilon_{r} \approx 80\right)$, mas são bem melhores que o valor que obtemos a partir da equação de Onsager (igualdade $(13)$ ), que é $\epsilon_{r} \approx 18,1$.

\section{Conclusão}

Neste artigo, guiamos o leitor ou a leitora à obtenção da equação de Kirkwood para fluidos polares puros de moléculas com polarizabilidades desprezíveis - a equação 38. No percurso, buscamos evidenciar ao máximo a semelhança entre o modelo de Kirkwood e o modelo de Onsager, aplicado a fluidos polares puros de moléculas com polarizabilidades desprezíveis. Pensamos que esta é uma contribuição pedagógica relevante, uma vez que o modelo de Kirkwood é apresentado, pelo mesmo [3], como uma extensão do modelo de Onsager, mas a semelhança entre os dois modelos é difícil de ser enxergada no artigo de Kirkwood.

\section{Agradecimentos}

Agradecemos à FACEPE pela bolsa de Iniciação Científica concedida a Adonias Barros, e ao professor Gustavo Camelo Neto, da UFPE, pela leitura crítica de uma versão inicial deste artigo.

\section{Referências}

[1] L. Onsager, Journal of the American Chemical Society 58, 1486 (1936).

[2] A. Barros e P. Peixoto, Revista Brasileira de Ensino de Física 42, e20200152 (2020).

[3] J.G. Kirkwood, The Journal of Chemical Physics 7, 911 (1939).

[4] H. Fröhlich, Theory of Dielectrics - Dielectric Constant and Dielectric Loss (Clarendon Press, Oxford, 1949).

[5] I.S. Oliveira, Física Moderna para iniciados, interessados e aficionados (Editora Livraria da Física, São Paulo, 2005), v. 1.

[6] J.D. Bernal e R.H. Fowler, The Journal of Chemical Physics 1, 515 (1933).

[7] C. Zhang, J. Hutter e M. Sprik, The Journal of Physical Chemistry Letters 7, 2696 (2016). 